\title{
Next WSN applications using ZigBee
}

\section{Xavier Carcelle ${ }^{1}$, Bob Heile ${ }^{2}$, Christian Chatellier $^{3}$, Patrick Pailler $^{4}$}

\author{
' Xavier Carcelle, IEEE Member, xavier.carcelle $(\omega$ ieec.org \\ 2 Bob Heile, ZigBee Alliance Chairman, bheile@iieee.org \\ 3 Christian Chatellier, University of Poitiers, France, chatellier@sic.sp2mi.univ- \\ poitiers.fr. \\ ${ }^{4}$ Patrick Pailler, University of Poitiers, France, Patrick.pailler@etu.univ-poitiers.fr
}

\begin{abstract}
Among the latest events of the wireless revolution, the fast-growing of ZigBee as a standard for WSN (Wireless Sensors Networks) is certainly one of these. ZigBee and 802.15.4 had been proving in the last years that they can achieve the results that Wi-Fi had achieved for high bit-rate wireless LANs and some large reliable deployments are now in place implementing ad-hoc WSN in critical applications. Therefore this paper will emphasize on the past, present and future features for ZigBee, taking a look on the feedback from previous implementations to finally design the next generations of WSN based on ZigBee.
\end{abstract}

Keywords: Wireless, Home Networking, Wireless Sensor Networks, ZigBee, IEEE 802.15.4. 


\section{Introduction}

One of this paper's aims is to give an up-to-date state-of-the-art concerning the WSN and their applications from a HAN (Home Area Network) point of view. WSN has been a hot topic lately with the development of embedded systems and the optimization of ad-hoc networks versus battery-friendly nodes. ZigBee has been definitely the most dominant wireless standard with the support of the IEEE 802.15 .4 working group since the version 1.0 in 2004 to the adoption of the protocol by some major industrial actors in 2007 . WSN are requiring reliable, batteryfriendly, secure, auto-configurable ad-hoc protocols and the second part of this article will give a view on the feedbacks of the first deployments using ZigBee. Then the article will emphasize on the use of ZigBee in the HAN applications showing possible cases for the positioning of ZigBee nodes. Finally the article will present an ideal plate-form based on several protocols and medium to get the best transmission for WSN using wired and wireless networks. 


\section{ZigBee past, present and next features}

ZigBee is now a well-known wireless standard within the landscape of IEEE since several years. The first proposal to IEEE was in late 2000 and the first specifications came out in 2003 for the MAC and PHY layer (from the IEEE 802.15.4) and in 2004 for the Network layer and remaining upper layers (from the ZigBee Alliance). 802.15.4 was revised in 2006 as were elements of the ZigBee to improve the functionalities, features and coexistence in the loaded 2.4 ISM band. Frequency Agility, network layer enhancements and added support for very large networks were added in 2007.

The figure 1 overviews the milestones of the development for the MAC and Network layer since the first initial MRD in 1999.

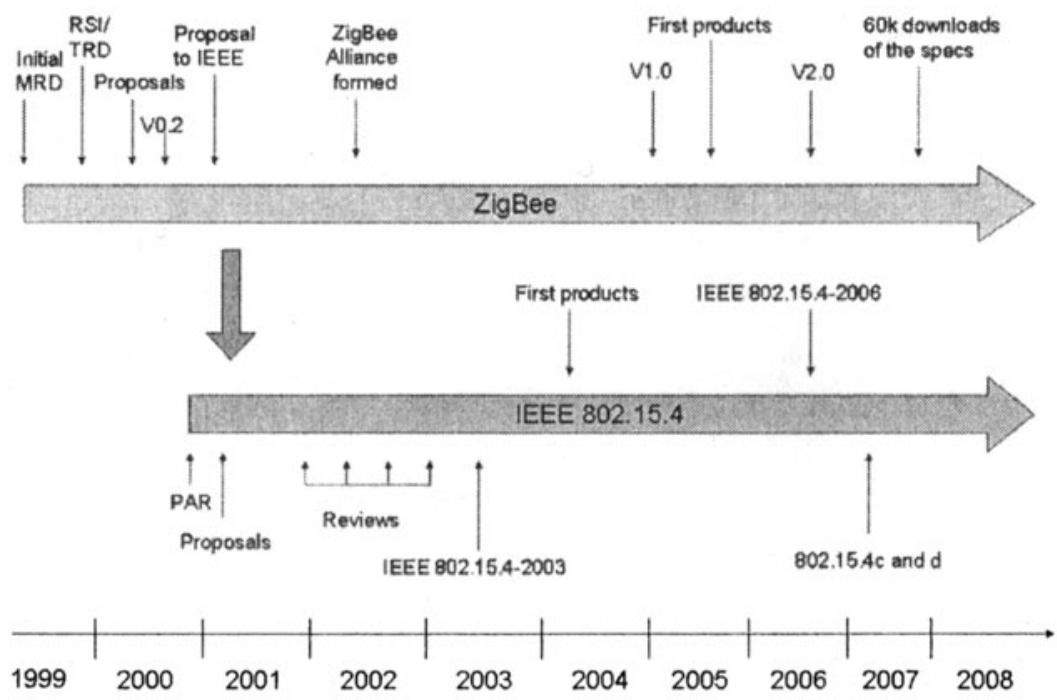

Figure 1: Milestones in the ZigBee history

Since the first products came out after the 1.0 version of the specifications of the ZigBee network stack, lots of ZigBee networks are now implemented worldwide in the WSN area such as the $4 \mathrm{k}$ nodes network in Korea, networks in Scandinavian sugar mills and North American hotels with ZigBee planned for deployment in $200 \mathrm{~K}$ electric meters in Gotenberg, Sweden. The past and current implementations are using extensively the ad-hoc features from the MAC (Medium Access Control) and network layer with the three types of nodes with such a network (ROUT for router, COORD for coordinator and END for end-device with reduced functionalities). 
Concerning the PHY (PHYsical) layer, ZigBee is based so far on two implementations: the "short-distance" one based on the ISM $2.4 \mathrm{GHz}$ band and the "longdistance" one based on the ISM $868 / 915 \mathrm{MHz}$ band. The specifications of these two bands are detailed in the table 1.15.4-2006 adds higher data rate modulations schemes to the sub $1 \mathrm{GHz}$ bands as well.

\begin{tabular}{|c|c|c|c|c|c|c|}
\hline \multirow{2}{*}{$\begin{array}{l}\text { PHY } \\
(\mathrm{MHz})\end{array}$} & \multirow{2}{*}{$\begin{array}{l}\text { Frequency } \\
\text { Band } \\
(\mathrm{MHz})\end{array}$} & \multicolumn{2}{|c|}{ Spreading parameters } & \multicolumn{3}{|c|}{ Data parameters } \\
\hline & & $\begin{array}{l}\text { Chip } \\
\text { rate } \\
\text { (kchip/s) }\end{array}$ & Modulation & $\begin{array}{l}\text { Bit rate } \\
\text { (Kbits/s) }\end{array}$ & $\begin{array}{l}\text { Symbol } \\
\text { rate } \\
(\mathrm{KSymb} / \mathrm{s})\end{array}$ & Symbols \\
\hline \multirow[t]{2}{*}{$868 / 915$} & $868-868.6$ & 300 & BPSK & 20 & 20 & Binary \\
\hline & $902-928$ & 600 & BPSK & 40 & 40 & Binary \\
\hline 2450 & $\begin{array}{l}2400- \\
2483.5\end{array}$ & 2000 & O-QPSK & 250 & 62.5 & $\begin{array}{l}\text { 16-ary } \\
\text { Orthogonal }\end{array}$ \\
\hline
\end{tabular}

Table 1: Frequency bands for ZigBee

In the past and current implementations, most of the chips available are based on the $2.4 \mathrm{GHz}$ bands due to the fact that this band allows devices to be sold anywhere in the world. For fixed equipment regional markets like outdoor metering networks, the sub $1 \mathrm{GHz}$ bands should start finding more interest. The IEEE 802.15.4 is currently releasing two new flavours available at the PHY layer: 802.15.4c for China $780 \mathrm{MHz}$ band and $802.15 .4 \mathrm{~d}$ for Japan $950 \mathrm{MHz}$ RFID band. These two new bands available for "long-distance" ZigBee will extend the possibilities of applications for outdoor implementations namely in those markets. The ZigBee eco-system is implement on top of the 802.15.4 MAC layer and the ZDO (ZigBee Device Object) allows the implementations of several ZigBee profiles targeting the appropriate applications (in-building automation, sensitive applications, mobile WSN...). The architecture is presented in the Figure 2. 


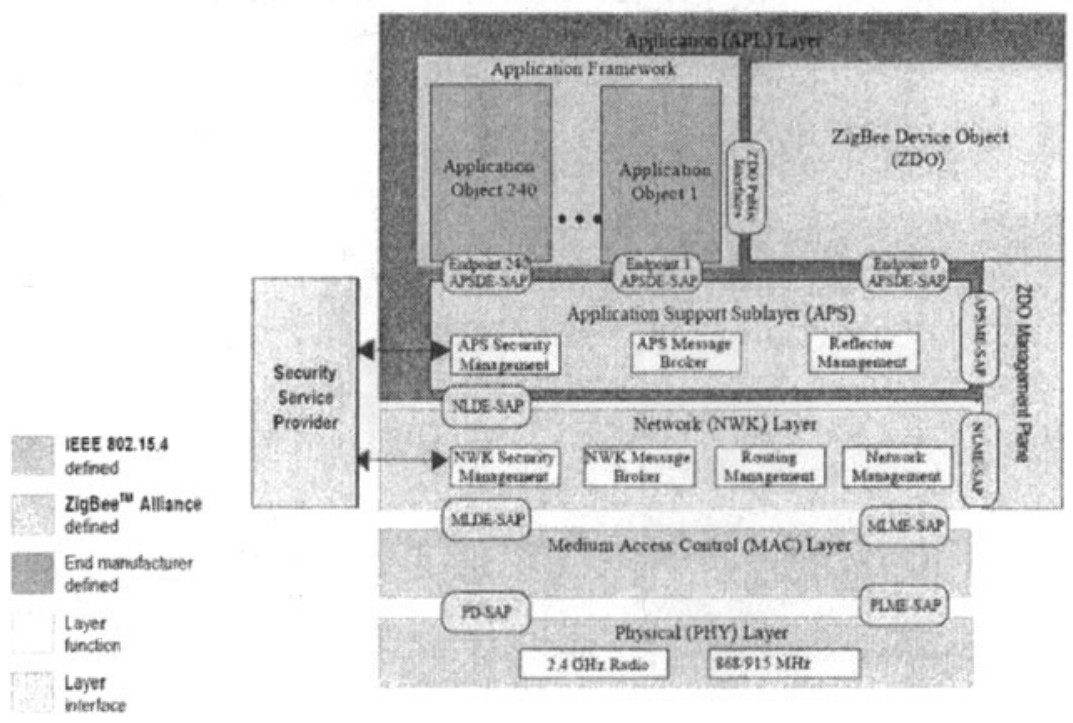

Figure 2: Architecture of the ZigBee stack

The ZigBee solution has been seen as a battery-friendly wireless standard for lowbit-rate, self-healing, mesh networks using its main strong features:

IEEE 64-bit or 16-bit network addresses for large WSNs

Flexibility of the configuration targeting the application (retransmission frequency, functions of each node on the net...)

Good implementation of the current ad-hoc functionalities

Flexible implementation of gateways to the outside world

The table 2 sums up the features of ZigBee as they can be seen from a timeline prospective 


\begin{tabular}{|c|c|c|c|c|}
\hline & & Past & Present & Future \\
\hline $\begin{array}{l}\text { ZigBee } \\
\text { tures }\end{array}$ & Fea- & $\begin{array}{l}\text { *868/916MHz pos- } \\
\text { sible usage } \\
{ }^{*} \text { Star topology pre- } \\
\text { ferred } \\
{ }^{*} \text { Fixed and small } \\
\text { WSN } \\
{ }^{*} \text { Development kits }\end{array}$ & $\begin{array}{l}{ }^{*} 2.4 \mathrm{GHz} \text { pre- } \\
\text { ferred for indoor } \\
\text { apps } \\
{ }^{*} \mathrm{Small} \text { mobile } \\
\text { WSN } \\
{ }^{*} \text { Large ad-hoc } \\
\text { fixed MSN } \\
{ }^{*} \text { Long distance } \\
\mathrm{ZB} \\
{ }^{*} \mathrm{Wi}-\mathrm{Fi} / \mathrm{ZB} \text { co- } \\
\text { habitation and } \\
\text { gateways } \\
{ }^{*} \mathrm{US} \text { Laws en- } \\
\text { forcement to } \\
\text { recognize ZB as } \\
\text { a WSN standard }\end{array}$ & $\begin{array}{l}\text { *Multiple homoge- } \\
\text { neous gateways to } \\
\text { others protocols } \\
{ }^{*} \text { Channel auto- } \\
\text { adaptation with the } \\
\text { new version } \\
{ }^{*} \text { Chinese/Japanese } \\
\text { ZB } \\
{ }^{*} \text { Embedded ZB ap- } \\
\text { plications } \\
{ }^{*} \text { ZB pro version }\end{array}$ \\
\hline
\end{tabular}

Table 2: ZigBee past, present and future features 


\section{Feedbacks on existing ZB applications}

Based on the existing WSNs deployed using ZigBee, there is a certain number of feedbacks available publicly on the performances and drawbacks of the current features. One of the issues in terms of design for ZigBee networks were the interference problems in the "busy" $2.4 \mathrm{GHz}$ frequency bands regarding the others technologies in the same ISM band. The 802.15.4 standard had been designed to keep some "immune" channels at the very edge of the 2.4 ISM band.

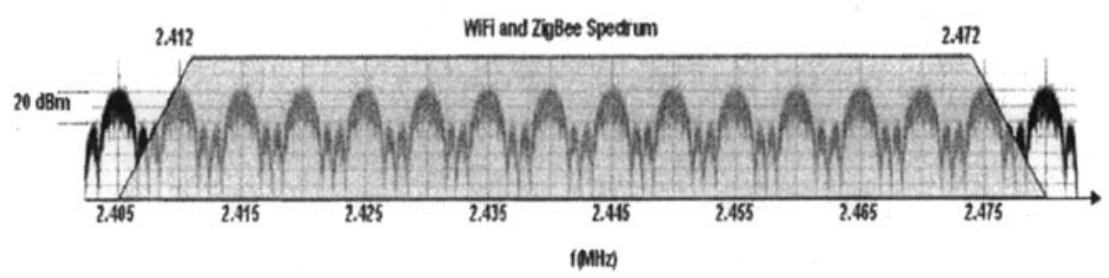

Figure 3: Wi-Fi and ZigBee spectrum

The figure 3 shows both the $802.11(\mathrm{~b} / \mathrm{g})$ spectrum and the 802.15 .416 channels and as we can see the channels 1 and 16 are actually out of the scope of the Wi-Fi spectrum. This is the same consideration for the 802.15.1 (BlueTooth at the network layer) interferences which remains in a narrower band than the 1 and 16 channels of ZigBee. Finally concerning the potential interferences coming from $2.4 \mathrm{GHz}$ microwaves, the duty cycle of the ovens keep a certain sufficient timeslot for the 802.15 .4 to be transmitted properly with not too many re-SYN packets. Measured ZigBee network performance in the presence of heavily loaded 802.11 networks has been very good as has been the performance for products implementing both technologies in the same box. This is true even for channels withing the 802.11 channel assignments.

Secondly the ZigBee had been seen also as wireless standard adjustable for the applications namely in the automation world. For instance the latency and the transmission frequency are not always depending on the applications. Therefore one can enable a certain number of options while deploying:

- Sleep and wake-up sensors for better battery life can be implemented with short duty timeslot as seen in figure 4 


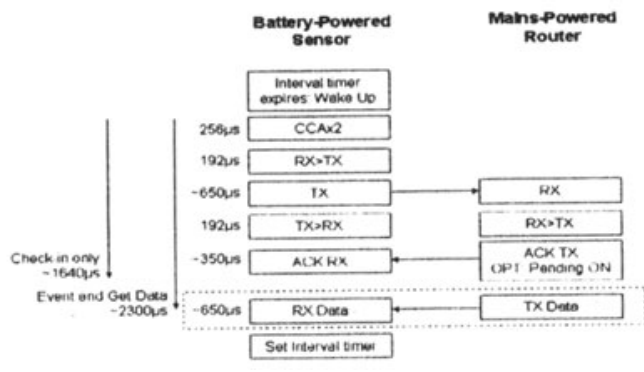

Figure 4: Sensor timing application (copyright ZigBee Alliance)

- Beacon-enabled or not ZigBee networking depending on the QoS (Quality of Service) requested on the deployed WSN. The figure 5 shows the communication to and from the COORD in the two cases and therefore the ACK (acknowledgment) can be optional.
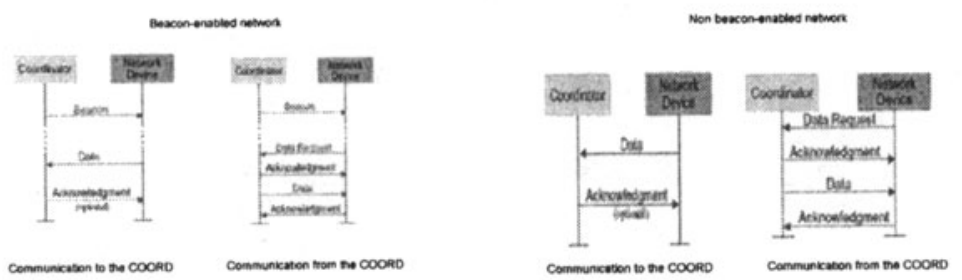

Figure 5: Transmission with the COORD with beacon frames enabled and not

- Long distance ZigBee for outdoor net-to-net uplinks. As shown in the figure 6 , some situations imply the deployment of WSN over several buildings while trying to have only large network (easier implementation, better monitoring, same on-top applications...). This can be achieved, for 
instance, by using ZigBec COORD nodes with an amplified RF interfaces (several $100 \mathrm{~mW}$ instead of the standardized few $\mathrm{mW}$ of the ZigBee specifications), this allowing reliable radio transmission over several hundreds meters outdoor up to one kilometer with a Line Of Sight configuration. The figure 6 presents an example of a unique WSN over a two-buildings topology.

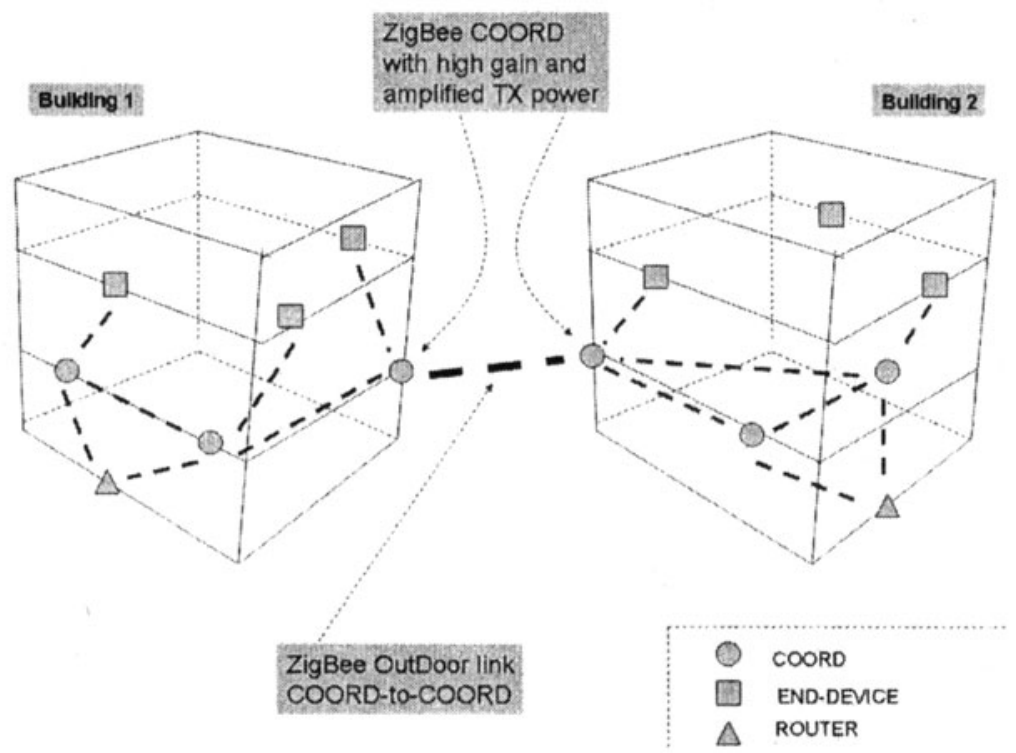

Figure 6: OutDoor two-buildings ZigBee applications

- Optimization of the data frame based on the targeted application. The figure 7 shows the lEEE 802.15.4 data frame (maximum size of 127o).

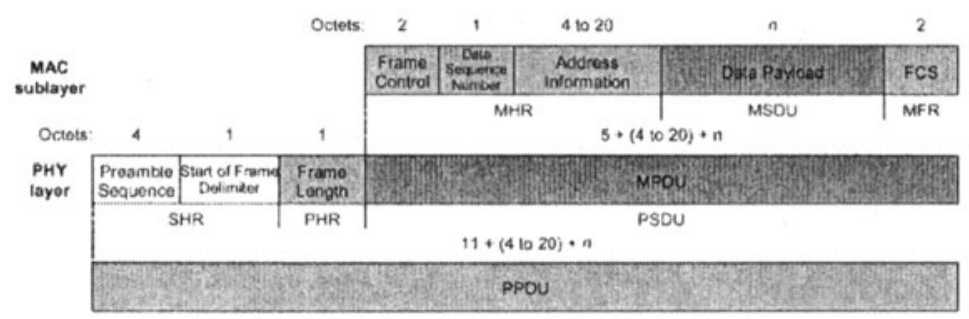

Figure 7 : Data frame format in IEEE 802.15.4 


\section{ZB, the best configurations for a HAN application}

\subsection{Positions of ROUTERS, COORD, ZED in the HAN}

At the MAC layer, the IEEE 802.15.4 standard specifies two types of devices (FFD for Full Function Device and RFD for Reduced Function Device) and at the network layer three type of devices (COORDINATORS, ROUTERS, and ZigBeeEndDevices) are defined by the ZigBee specifications. A Coordinator device is the same as a Router device except for its role in the network. Basically it is a Router that has acted as the leader in network formation. Each network has one Coordinator. A Router is able to forward packets and participate in the mesh network. A ZED can only send a receive packets intended for it. Ideally all powered devices should be Router devices allowing the device to be a node of the ad-hoc network. In a real case it should be appropriate to have ZEDs for battery powered devices anywhere in the network and the remote sensor nodes at the network edges (such as upper floors, corners, hidden parts, ceiling...) and relay through Router devices placed in several central positions in-house. As long as there are a reasonable number of Router devices distributed around the building in powered applications like load controllers and lights, ZEDs can be anywhere in the network. For "thin" networks it is better to have then near the edges.

\subsection{The Smart-House case}

The figure 8 presents the illustration of a large house equipped with a high number of sensors allowing several applications based on those. This smart house is equipped with different type of sensors (temperature, heat alarms, gas, time controls, pressure, HVAC controls...) spread out all over the different floors, indoor and outdoor. 


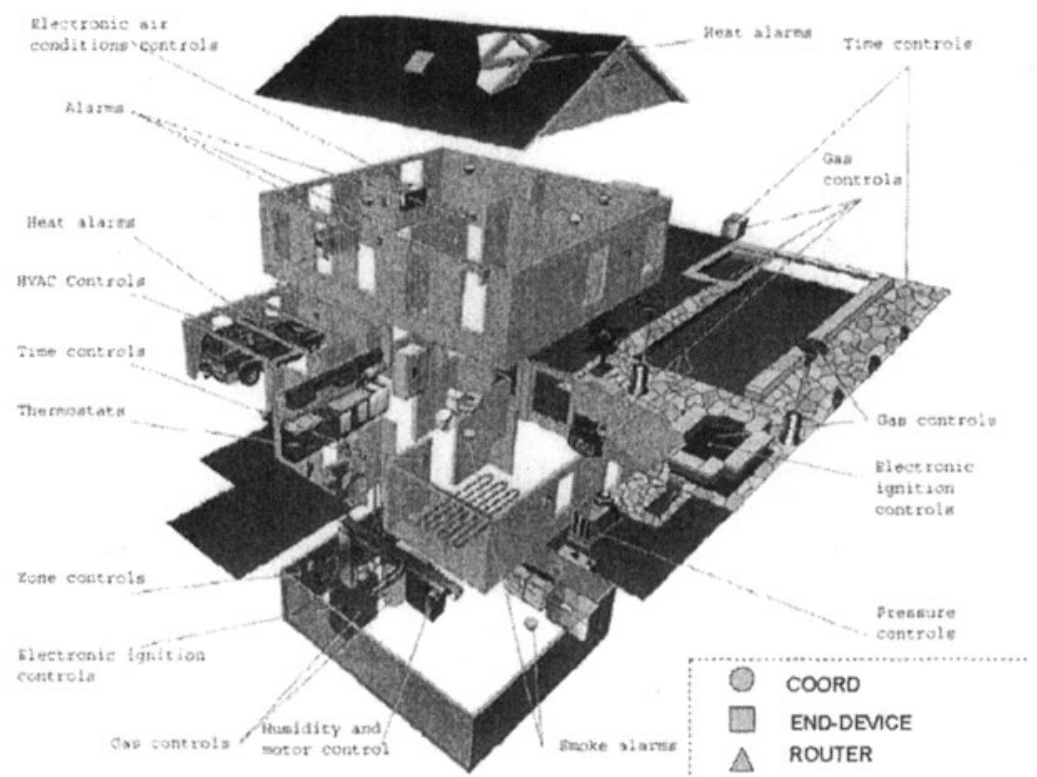

Figure 8: The ZB smart house

Based on the design of this smart house, each ZigBee device should be place at the best position, with the best functionalities to improve the all WSN and allow battery-friendly applications to retrieve the data from each sensor with a reliable wireless link. The figure 9 represents the same design of smart house with the frames of each floor allowing a better identification of the best position for each node. 


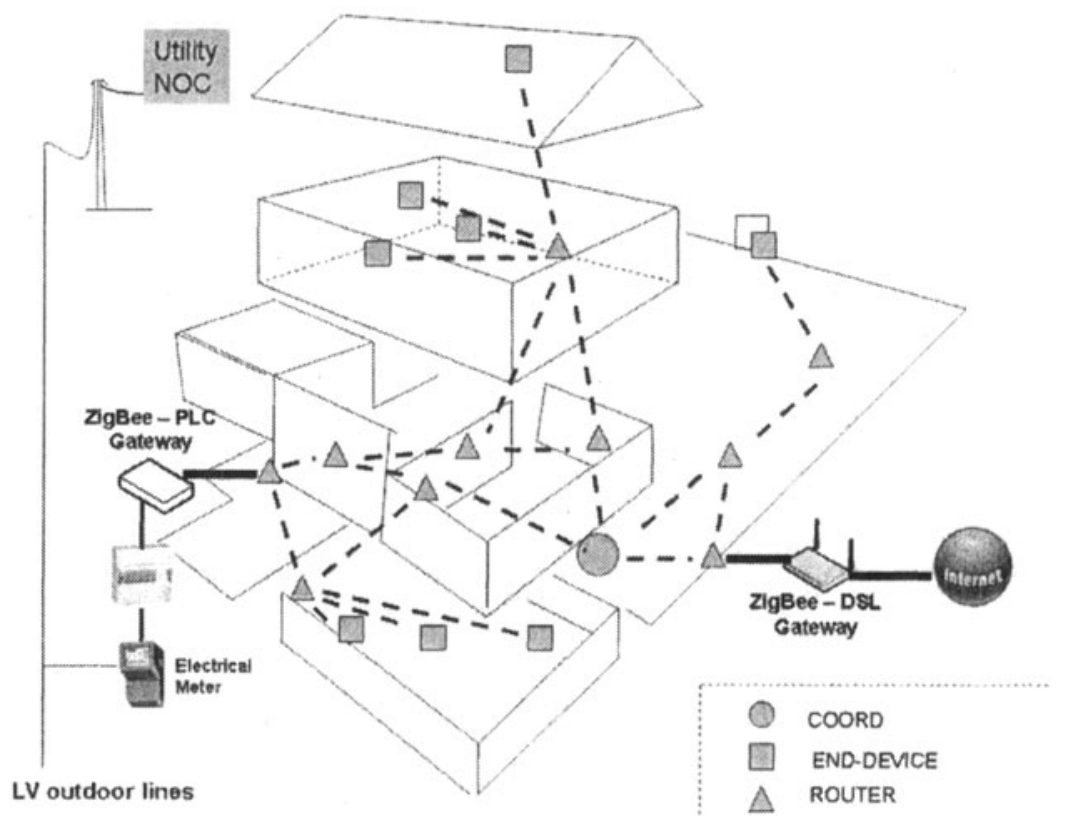

Figure 9: ZB Smart-House - Positions of the devices

Most of the remote nodes (high floors, far-end of the garden, basement, ceiling...) can be implemented as ZEDs allowing a better battery life and no routing functionalities. The ZEDs connect to a nearby Router in a star topology. Then the central nodes (mid-floors, central rooms...) should be implemented where possible as Routers to allow a mesh network relaying the data from the ZEDs to the end of the network. The different Router devices form a mesh topology where each node relay the data and update its routing table based on the IETF-certified protocol AODV. Finally, the Router devices are connected to one or several ROUT devices that are part of the gateways. In this example, two gateways are shown:

- A ZigBee-to-DSL gateway. This gateway allows the connexion of the WSN to Internet, therefore to the online applications to retrieve, monitor and control the sensors offsite.

- A ZigBee-to-PLC gateway. In this example, we consider that the electrical utility has implemented a PLC access network enabling the IP connexion from each house (each meter in each house) the backhaul of the utility and to the NOC (Network Operation Center). 


\subsection{Battery considerations}

During the design and deployment of a WSN, the questions of battery are very important and should be addressed by using the best features from the wireless technology selected. The table 3 shows a comparison between two PAN (Personal Area Network) technologies in the IEEE 802.15 working group. The ZigBee standard has been designed to prevent the problem of heavy power cycle based on the best RF transmission modulation technique, the optimization of the SYN/ACK methods and the low data rate allowing the transmission of small PPDU.

\begin{tabular}{|l|l|l|l|l|}
\hline Standard & Modulation & $\begin{array}{l}\text { Max data } \\
\text { rate }\end{array}$ & $\begin{array}{l}\text { Sensitivity of } \\
\text { the interface }\end{array}$ & Duty cycle \\
\hline ZigBee & $\begin{array}{l}\text { OQPSK with } \\
\text { shaping }\end{array}$ & $128 \mathrm{Kbits} / \mathrm{s}$ & $\begin{array}{l}-90 \mathrm{dBm} \\
40 \mathrm{ppm} \text { xtal }\end{array}$ & Extremely low \\
\hline Bluetooth & FSK & $720 \mathrm{Kbits} / \mathrm{s}$ & $\begin{array}{l}-85 \mathrm{dBm} \\
20 \mathrm{ppm} \text { xtal }\end{array}$ & $\begin{array}{l}\text { Cycled power } \\
\text { applications } \\
\text { (handsets, } \\
\text { cellphones...) }\end{array}$ \\
\hline
\end{tabular}

Table 3: Battery considerations with a comparison between ZigBee and BlueTooth

This considerations lead to think the positioning of each node in a smart house as for example:

- ZigBee ZED nodes: sensors with long-battery life, specific wake-up-onbeacon activation enable and long retransmission period (based on the needs of retransmitting the data of the sensors)

- ZigBee COOR/ROUTER nodes: central sensors (positioned centrally in the house) with mesh-routing enable and powered directly to the powerline of the house.

- ZigBee Gateways: positioned next to an external network connection (connecting the WSN to the outside world) such as a DSL/Cable/WiMAX Internet-box or next to the circuit breakers box. In a large network, it may be necessary to have more than one gateway. These should be distributed around the network. 


\section{A potential ideal platform for $H A N$}

The different considerations developed here in this article lead to think the nextgeneration of WSN as a ZigBee large network coupled with different others protocols to allow a better radio transmission and a pervasive connectivity. The recent co-developments of ZigBee with different others network protocols imply in a close-future all-in-one gateways from ZigBee to others standard protocols for WSN or for Ethernet-based network.

\subsection{Embedding ZigBee}

The next generation of products based on ZigBee will help to build embedded ZigBee systems. These embedded systems will allow an easier design of a WSN with the possibility of using cheaper and standard OS (Operating System) with access to the ZigBee/802.15.4 stack. Lately the next generation of ZigBee products had been based on:

- New Soc (Systems on Chip)

- PCMCIA/USB pccards

- ZigBee female power plugs

- OEM ZigBee set-top-boxes

\subsection{Gateways to the wired standard protocols}

In the scope of the WSN protocols, the wired protocols are still seen as more reliable, stable and easy to connect to the standard networks media (Ethernet, FTP cable, coax, twisted pairs, powerline cables...). Therefore the ZigBee had been developing better interfaces between the ZigBee stack (and the 802.15.4 frames) and different others Home Automation protocols

\begin{tabular}{|l|l|}
\hline Standards & Functionalities \\
\hline ZigBee and BACNet & $\begin{array}{l}\text { Building Automation and Control Networks based on } \\
\text { the ISO-16484-5 with messages-based protocols. } \\
\text { Classes of applications services for Home Automation } \\
\text { and Object Access Services. Ethernet LAN options. } \\
\text { "BACNet/IP"-like encapsulation of BACNet messages } \\
\text { in 802.15.4 frames. }\end{array}$ \\
\hline ZigBee and DALI & $\begin{array}{l}\text { Digital Addressable Lighting Interface based on the } \\
\text { IEC 62386 lighting control systems. Uses ballasts, } \\
\text { transformers, actuators from multiple manufacturers in } \\
\text { the one installation. Up to 64 DALI light sources. Ca- } \\
\text { bling consists of a simple two wire cable }\end{array}$ \\
\hline ZigBee and Lonworks & The LonWorks protocol can be implemented over ba- \\
\hline
\end{tabular}




\begin{tabular}{|l|l|}
\hline & $\begin{array}{l}\text { sically any medium, including power line, twisted pair, } \\
\text { radio frequency (RF), infrared (IR), coaxial cable and } \\
\text { fiber optics. Therefore a LonWorks encapsulated into a } \\
\text { ZigBee network can be designed as well as a ZigBee } \\
\text { large WSN including LonWorks-to-ZB sensor nodes. }\end{array}$ \\
\hline ZigBee and HomePlug & $\begin{array}{l}\text { HomePlug is from a market point of view the PLC } \\
\text { worldwide standard allowing the design of Ethernet } \\
\text { network over the electrical wiring of a building. There- } \\
\text { fore ZigBee nodes can be connected to the wireless } \\
\text { eco-system but also to PLC gateways allowing the } \\
802.15 .4 \text { frames to be encapsulated into Ethernet } \\
\text { frames (802.3). Finally this network then can be seen } \\
\text { as a ZigBee network using a PLC backhaul. }\end{array}$ \\
\hline
\end{tabular}

Table 4: ZigBee to other gateways functionalities

\subsection{Up-grading to the newer version}

The different ZB nodes of the WSN are now based on different vendors SoC implementing the specifications from the ZigBee Alliance and from the IEEE 802.15.4 working group. These Soc are then based on the features from the ZB specifications such as remote commands at the MAC Layer. Therefore the ZB chip can be upgraded on-the-fly from a central point of the network without loosing the applications layer and the services on top of that. The next generation of WSN will allow a complete pervasive topology where from any IP network address, all nodes from the WSN will addressed and then flashable with the latest specifications and optimization.

\section{Conclusion}

The next generation of WSN will enjoy the coming features of standardized wireless protocols like ZigBee which seams to be seen as the "Wi-Fi of the sensors". ZigBee is a battery-friendly protocol, able to implement a mesh network for up-to 2000 nodes as seen in different industrial applications. Moreover the next ZigBee specifications are covering the interfaces with different other Home Automation protocols to design a complete WSN using wireless and wired interfaces. The last deployment using ZigBee had been proving reliability sufficient to be applied for sensitive applications by implementing the correct retransmission process, error correction algorithm and radio link quality estimation.

The next generation WSN can be seen as a hybrid network (wired/wireless, IP-toZigBee encapsulation, multiple gateways...) with different connexions to the outside world of IP, different levels of services, different ways of monitoring (push 
from the public network, get from the end-users, alarms-based messages sending, online HTTP interfaces...) where the IP networking is prolonged to the last nodes of the WSN.

\section{References}

[1] J.Y. Chen, X.P. Zhou, "ZigBee Wireless Communication Technology in Industrial Controls," Radio Engineering of China, Vol. 36, No. 6, pp.

61-64, 2006.

[2] G. Pekhteryev, Z. Sahinoglu[P. Orlik, G. Bhatti, « Image transmission over IEEE 802.15.4 and ZigBee networks " Proceedings of ISCAS 2005. Japan: Kobe $\square 2005$, pp. 3539-3542.

[3] W.Y. Zhang, L. Feng, Z.C. Wei, "Research on home networking with ZigBee," Journal of Hefei University of Technology(Natural Science), Vol. 28, No. 7, pp. 755-799, July 2005.

[4] P.G. Feng, L. Feng, Z.C. Wei, "An Application Model in Home Network Based on Zigbee," Journal of Henan University of Science \& Techn

[5] R. Yang, T. Yang, "Wireless Sensor Network Based on ZigBee with Applications for Smart Building," Agricultural mechanization of China,

Vol. 5, pp. 76-79, May 2005.

[6] P Baronti, P Pillai, Vince W.C. Chook, S Chessa, AGotta, Y. Fun Hu, Wireless sensor networks: A survey on the state of the art and the

802.15.4 and ZigBee standards, Computer Communications 30 (2007) 1655-1695

[7] I F Akyildiz, X Wang, W Wang, "Wireless mesh networks: a survey", Computer Networks 47 (2005) 445-487 